\title{
Atopische Dermatitis
}

\section{Cremen gegen den „allergischen Marsch“}

\begin{abstract}
Bei Neugeborenen mit erhöhtem Atopierisiko könnte eine konsequente Basistherapie mit geeigneten Externa möglicherweise die Manifestation einer atopischen Dermatitis verhindern und damit frühzeitig dem Beginn des „allergischen Marschs" begegnen.
\end{abstract}

Diese Hoffnung wecken nun jedenfalls die Ergebnisse zweier aktueller Studien, die Prof. Dr. Thomas Werfel von der Medizinischen Hochschule Hannover auf dem 10. Deutschen Allergiekongress vorgestellt hat.

Im Rahmen einer in England und den Vereinigten Staaten durchgeführten Studie waren insgesamt 124 Neugeborene aus Risikofamilien für atopische Erkrankungen ab der dritten Lebenswoche randomisiert bis zum sechsten Lebensmonat mit einer auf den gesamten Körper aufgetragenen wirkstofffreien Creme behandelt worden oder blieben unbehandelt. Allein durch diese Maßnahme ließ sich das kumulative Risiko für die Entwicklung einer atopischen Dermatitis um 50\% senken, berichtete Werfel.

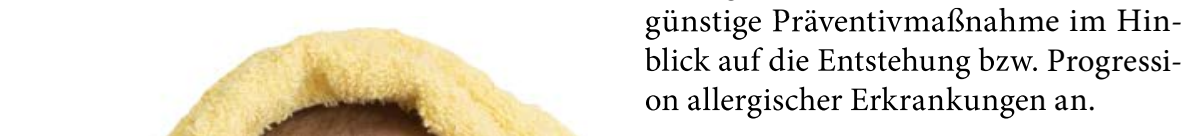

Kann einfache Basiscreme einen Schutzmantel gegen Neurodermitis-Ausbrüche aufbauen?

\section{Basistherapie als kostengünstige Präventivmaßnahme}

Eine Bestätigung finden diese Daten in einer ähnlich konzipierten japanischen Untersuchung. Auch in dieser Studie wurden aus Risikofamilien stammende Neugeborene, bei denen mindestens ein Elternteil oder Geschwister an einer atopischen Dermatitis erkrankt war, mit einer wirkstofffreien Basiscreme behandelt - in diesem Fall bis zur 32. Lebenswoche. Gegenüber unbehandelten Kindern ließ sich auch in dieser Studie die Inzidenz der atopischen Dermatitis um 32\% senken. Ein Effekt auf die Hühnerei-spezifische IgEProduktion ließ sich nicht feststellen.

Sollten sich diese nach Einschätzung Werfels nahezu sensationellen Daten in größeren Studien bestätigen lassen, böte sich eine bei Risikokindern konsequent durchgeführte Basistherapie als kostengünstige Präventivmaßnahme im Hinon allergischer Erkrankungen an.

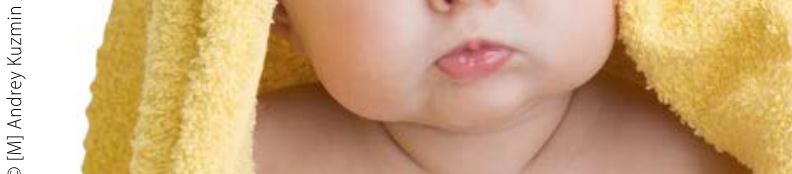

Protektive Effekte einer entsprechenden Basistherapie ließen sich möglicherweise auch durch günstige Effekte auf die intakte Hautbarriere erklären. Tatsächlich gibt es nach Darstellung Werfels inzwischen klare Hinweise darauf, dass sich Patienten mit atopischer Dermatitis über die Haut auch eine Sensibilisierung gegenüber Nahrungsmitteln einfangen können - ein Grund mehr, gemäß den Empfehlungen der aktualisierten Neurodermitis-Leitlinie bei der Diagnostik und Therapie nicht nur auf die Haut zu achten, sondern auch typische Komorbiditäten wie Nahrungsmittelallergien, Asthma oder allergische Rhinitis im Auge zu halten.

\section{Sensibilisierung gegen \\ Nahrungsmittel über die Haut}

Die Möglichkeit einer Sensibilisierung gegen Nahrungsmittel über die Haut legen nun auch Ergebnisse einer britischen Untersuchung nahe, die bei 619 ausschließlich gestillten Säuglingen nach dem dritten Lebensmonat ein mehr als sechsfach erhöhtes Risiko für eine Sensibilisierung gegenüber Nahrungsmittelallergenen fand, wenn diese Kinder an einer atopischen Dermatitis erkrankt waren. Zudem fand sich eine klare Korrelation zwischen dieser Sensibilisierung und dem Schweregrad der atopischen Dermatitis.

Möglich wäre eine über die Haut entstehende Sensibilisierung gegen Nahrungsmittel etwa durch Hautkontakt mit Fremdproteinen, wie z. B. mit Erdnussproteinen. Neben hohen Raumluftkonzentrationen wäre eine Sensibilisierung auch über Pflegeprodukte oder Badezusätze, die man bei Neurodermitikern zurückhaltend oder am besten gar nicht einsetzen sollte, möglich. Gleiches gilt nach Empfehlung Werfels auch für den Zusatz von Kuhmilchproteinen im sog. Kleopatrabad. (Ludger Riem)

Literatur beim Verlag;

Werfel T, Dermatologie - Allergologie aktuell.

AllergoCompact: Update Neurodermitis. 10. Deutscher Allergiekongress, Köln 2015 